\title{
Württemberg, Tübingen und die Reformation in Südosteuropa
}

\author{
von Franz BREndLE
}

\section{Die Reformation und Europa}

Das Reformationsjubiläum 2017 gibt auch dazu Anlass, der Bedeutung der von Wittenberg ausgehenden Reformation für den europäischen Osten nachzugehen. Die Reformation breitete sich in den 1520er Jahren nicht nur innerhalb des Reichs schnell aus, sondern auch jenseits seiner Grenzen, vor allem in jenen Territorien, die durch die Siedlungsbewegungen des Mittelalters im Einflussbereich der deutschsprachigen Kultur standen, auch wenn die Formierungsphase der reformatorischen Konfessionskirchen im östlichen Europa länger dauerte als im Reich. Dabei kann gerade für die Reformationsentwicklung Südosteuropas ein spezifisch württembergischer Einfluss geltend gemacht werden, der sich in erster Linie mit dem Namen Primus Truber verbindet. ${ }^{1}$ Der Geist weht zwar, wo er will, aber er bewegt sich doch gern auf vorgegebenen politischen Bahnen. Und das war die kleinteilige territoriale Welt des deutschen Südwestens mit ihren zahlreichen kleinen und kleinsten Herrschaften, wie sie eine typische Regionallandschaft des Alten Reiches kennzeichnen. ${ }^{2}$ So ist es naheliegend, zunächst die Reformation im Südwesten in den Blick zu nehmen, um von hier aus auch die daraus resultierenden Entwicklungen zu betrachten. ${ }^{3}$

1 Dazu neuerdings der große Sammelband: Sönke Lorenz u. a. (Hg.): Primus Truber 1508-1586. Der slowenische Reformator und Württemberg. Stuttgart 2011.

2 Volker Press: Die territoriale Welt Südwestdeutschlands 1450-1650. In: Die Renaissance im deutschen Südwesten. Katalog zur Ausstellung des Landes Baden-Württemberg in Heidelberg. Karlsruhe 1986, S. 17-61; Ders.: Die Territorialstruktur des Reiches und die Reformation. In: Rainer Postel, Franklin Kopitzsch (Hg.): Reformation und Revolution. Beiträge zum politischen Wandel und den sozialen Kräften am Beginn der Neuzeit. Festschrift für Rainer Wohlfeil zum 60. Geburtstag. Stuttgart 1989, S. 239-268.

3 Zu Württemberg im 16. Jahrhundert vgl. Franz Brendle: Das Herzogtum Württemberg im 16. Jahrhundert. Land und Regenten im Zeichen von Herrschaftskrise, Reformation und Luthertum. In: Sönke Lorenz u. a. (Hg.): Vom Schüler der Burse zum „Lehrer Deutschlands“. Philipp Melanchthon in Tübingen. Tübingen 2010, S. 51-69; Ders.: Württemberg 1534-1568: Restitution, Reformation, lutherischer Landesstaat. In: Götz Adriani, Andreas Schmauder (Hg.): 1514 - Macht, Gewalt, Freiheit. Der Vertrag zu Tübingen in Zeiten des Umbruchs. Tübingen 2014. 


\section{Die württembergische Historiographie und die Herzöge}

Als zu Beginn des 20. Jahrhunderts die Amanduskirche in Bad Urach restauriert wurde, fanden auch die Konsolen an den Mittelschiffpfeilern, auf denen seit 1520 die Kirchenpatrone Maria und Amandus gethront hatten, eine neue Gestaltung. ${ }^{4}$ Nach dem Uracher Bildertag 1537 waren nämlich die Plastiken der Kirchenpatrone entfernt worden, lediglich die beiden Konsolen hatten den Bildersturm überlebt und waren stehen geblieben. Bei der Neugestaltung sollten nun die für das evangelische Württemberg wichtigen Herrscher als neue „Säulenheilige" quasi den Platz der alten Kirchenpatrone einnehmen. Als diejenigen Fürsten, die entscheidend für die Reformation in Württemberg eingetreten waren, wären vor allem Herzog Ulrich und Herzog Christoph in Frage gekommen. Ersterer hatte die Reformation eingeführt, letzterer Württemberg zu einem lutherischen Musterstaat ausgebaut. Und so trägt nun die eine Konsole seit 1905 die Figur des neuen „Kirchenpatrons“ Herzog Christoph, obwohl die Inschrift Sanctus Amandus, huius ecclesiae patronus, bis heute auf die ursprüngliche Funktion hinweist. ${ }^{5}$ Schwieriger tat man sich offensichtlich mit der Auswahl des zweiten Fürsten. Letztlich wurde Herzog Eberhard im Bart ausgewählt, der zwar schon lange vor der Reformation gestorben war, aber wohl doch besser in das Anforderungsprofil für einen Platz in der Kirche passte.

Herzog Ulrich von Württemberg wurde nicht berücksichtigt. Er, der die Reformation in Württemberg erst durchgesetzt hatte, schien den Urachern denn doch nicht als ein Fürst geeignet, den man in einer Kirche auf den Sockel stellen wollte. Dazu haben die persönlichen und politischen Verfehlungen des Herzogs sicher ein gerüttelt Maß beigetragen. Bereits vielen Zeitgenossen war Herzog Ulrich von Württemberg ein zutiefst verhasster Fürst. Als großen Tyrannisierer, als der wilden Säu Vater, so hat Dionysius Dreytwein, der Esslinger Chronist, Herzog Ulrich von Württemberg einmal beschimpft. ${ }^{6}$ Bei dieser Beurteilung spielten nicht nur die persönlichen Verfehlungen Herzog Ulrichs eine Rolle, sondern auch das so unnachbarschaftliche Verhältnis zu den schwäbischen Reichsstädten, zumal Ulrich gerade mit Esslingen immer wieder in harten Auseinandersetzungen stand. Ulrich war einer, dem man so ziemlich alles zutraute, selbst dasjenige, wofür er erwiesenermaßen nichts konnte. Und selbst seine eigenen Bundesgenossen, wie etwa Landgraf Philipp von Hessen, dem Ulrich tatsächlich

4 Das Folgende nach: Franz Brendle: Ulrich von Württemberg (1487-1550). In: Susan Richter, Armin Kohnle (Hg.): Herrschaft und Glaubenswechsel. Die Fürstenreformation im Reich und in Europa in 28 Biographien. Heidelberg 2016, S. 146-163.

5 Franz Brendle: Dynastie, Reich und Reformation. Die württembergischen Herzöge Ulrich und Christoph, die Habsburger und Frankreich. Stuttgart 1998, S. 346 f.

6 Adolf Diehl (Hg.): Dionysius Dreytweins Esslingische Chronik (1548-1564). Tübingen 1901, S. $74 \mathrm{f}$. 
zu großem Dank verpflichtet war, hatten es in ihrem Umgang mit dem ,Alten von Württemberg" nicht immer leicht. ${ }^{7}$

Die Geschichtsschreibung hat sich mit Herzog Ulrich von Württemberg ebenso schwer getan. ${ }^{8}$ Nicht einmal die württembergischen Hofhistoriographen vermochten es, ihn als einen Sympathieträger des Landes zu zeichnen. Tunlichst hat man von dieser Seite darauf verzichtet, die schlimmsten Exzesse Ulrichs darzustellen oder hat sie zumindest kaschiert. ${ }^{9}$ Seine politische Unerfahrenheit wurde ins Feld geführt, die Fehler seiner Gegner hervorgehoben. So entstand eine zum Teil merkwürdig anmutende Apologie Ulrichs, die im 19. Jahrhundert geradezu in einer romantischen Verklärung des Herzogs gipfelte. Er wurde jetzt zum Bürgerkönig stilisiert, den man sich in der Zeit so sehr erhoffte. Ähnlich wie Eberhard im Barte wurde Ulrichs Umgang mit seinen Untertanen als so vertrauensvoll beschrieben, dass er jedermann sein Haupt in den Schoß legen konnte. Der Roman Lichtenstein von Wilhelm Hauff, der bis heute in weiten Teilen der Bevölkerung als historisches Zeugnis dieser für Württemberg schicksalshaften Zeit gelesen wird, ist ein herausragendes Zeugnis jener Umdeutung. Die Geschichte des vertriebenen Herzogs, der sich in Sehnsucht nach Land und Leuten verzehrt, heimlich des Nachts im Land Unterschlupf sucht, dort von treuen Anhängern auf dem Lichtenstein versorgt wird, angesichts seiner aussichtslosen Lage seine Vergehen bereut und es schließlich doch noch mit Gottes Hilfe und seiner treuen Württemberger erreicht, in die angestammte Herrschaft zurückzukehren - dieses Bild hat sich tief ins historische Bewusstsein eingeprägt. So sehr, dass die Burg Lichtenstein im 19. Jahrhundert wiedererrichtet wurde und namhafte Historiker

7 Zu Philipp von Hessen vgl. Inge Auerbach (Hg.): Reformation und Landesherrschaft. Vorträge des Kongresses anlässlich des 500. Geburtstages des Landgrafen Philipp des Großmütigen von Hessen vom 10. bis 13. November 2004 in Marburg. Marburg 2005; Ursula Braasch-Schwersmann u.a. (Hg.): Landgraf Philipp der Großmütige 1504-1567. Hessen im Zentrum der Reform, Begleitband zu einer Ausstellung des Landes Hessen. Marburg/Neustadt a. d. Aisch 2004; Wolfgang Breul, Holger Th. Gräf: Fürst, Reformation, Land - Aktuelle Forschungen zu Landgraf Philipp von Hessen (1504-1567). In: Archiv für Reformationsgeschichte 98 (2007), S. 274-300; Volker Press: Landgraf Philipp der Großmütige von Hessen. In: Klaus Scholder, Dieter Kleinmann (Hg.): Protestanten. Von Martin Luther bis Dietrich Bonhoeffer. Frankfurt am Main ${ }^{2} 1992$, S. 60-77; Gabriele Haug-Moritz: Reich und Konfessionsdissens im Reformationszeitalter. Überlegungen zur Reichskonfessionspolitik Landgraf Philipps des Großmütigen von Hessen. In: Hessisches Jahrbuch für Landesgeschichte 46 (1996), S. 137-159; Walter Heinemeyer: Philipp der Großmütige und die Reformation in Hessen. Gesammelte Aufsätze zur hessischen Reformationsgeschichte, hg. von Hans-Peter Lachmann. Marburg 1997.

8 Zu Herzog Ulrich: Brendle, Dynastie (wie Anm. 5); Ders.: Herzog Ulrich - ein verkannter Reformationsfürst. In: Siegfried Hermle (Hg.): Reformationsgeschichte Württembergs in Porträts, Holzgerlingen 1999, S. 199-225; Ders.: Ulrich, Hzg. v. Württemberg. In: LThK 10 (2000), Sp. 359-360; Horst Carl: Ulrich. In: Sönke Lorenz u. a. (Hg.): Das Haus Württemberg. Ein biographisches Lexikon. Stuttgart 1997, S. 103-106; Ludwig Friedrich Heyd: Ulrich Herzog zu Württemberg. Ein Beitrag zur Geschichte Württembergs und des deutschen Reiches im Zeitalter der Reformation, 3 Bde. Tübingen 1841/44; Volker Press: Herzog Ulrich (1498-1550). In: Robert Uhland (Hg.): 900 Jahre Haus Württemberg. Leben und Leistung für Land und Volk. Stuttgart ${ }^{3} 1985$, S. 110-135.

9 Vgl. dazu auch Brendle, Dynastie (wie Anm. 5). 
zu Beginn des 20. Jahrhunderts ernsthaft darüber diskutierten, welche Höhle auf der Schwäbischen Alb vom Herzog als Versteck genutzt wurde.

Wenngleich die Akten ein gänzlich anderes Geschehen zeichnen, nämlich das eines gehetzten Exulanten, der es niemals hätte wagen können und es auch niemals gewagt hat, nicht einmal heimlich ins Land zurückzukehren, weil ihm die habsburgischen Häscher selbst in Mömpelgard auf den Fersen waren und ihm nach dem Leben trachteten, so enthält der Lichtenstein-Roman doch den Kern einer historischen Rechtfertigung, die man seit der zweiten Hälfte des 16. Jahrhunderts in Bezug auf Herzog Ulrich vorgenommen hat. Dass er nämlich durch das Exil geläutert und erst dadurch fähig geworden sei, seiner eigentlichen Lebensaufgabe nachzukommen und das Land der Reformation zuzuführen. Diese in ihrem eigentlichen Sinn geistliche Aufgabe konnte nur von einem Herrscher wahrgenommen werden, der die Schuld seiner Jugendjahre gebüßt hatte und damit von Gott zu einem größeren Werk herangezogen werden konnte. Das war in den Augen der württembergischen Historiographie die historische Lebensleistung des Herzogs. Und auf diesem Hintergrund konnte man auch die Zeit der ersten Regentschaft bis zu einem gewissen Maße durchaus kritisch hinterfragen, zumal sich damit der Kontrast zum „späten Ulrich“ umso deutlicher herausarbeiten ließ. Dabei wurde man nicht müde, auf die schwierigen persönlichen und politischen Begleitumstände seiner Regierungsübernahme hinzuweisen.

\section{Der „maßlose“ Herzog: Ulrich von Württemberg}

Der unter misslichen familiären Umständen aufgewachsene Ulrich gelangte 1503 bereits vorzeitig an die Regierung, die von ausgesuchter höfischer Pracht und politischem Eigensinn gekennzeichnet war. Um den aus dem steigenden Steuerdruck resultierenden Bauernaufstand des „Armen Konrad“ niederwerfen zu können, verbündete sich Ulrich mit den Landständen, denen er im Tübinger Vertrag 1514 bedeutende Mitspracherechte im Herzogtum zugestehen musste. ${ }^{10}$ Die Ermordung seines adeligen Dieners Hans von Hutten und das Scheitern der 1511 mit Sabina von Bayern geschlossenen Ehe führten den Herzog in die politische Isolation und in die Reichsacht. ${ }^{11}$ Nach Ulrichs Überfall auf die Reichsstadt Reutlingen wurde er 1519 vom Schwäbischen Bund aus seinem Territorium vertrieben, das Land gegen die Erstattung der Kriegskosten an Karl V.

10 Andreas Schmauder: Württemberg im Aufstand. Der Arme Konrad 1514. Ein Beitrag zum bäuerlichen und städtischen Widerstand im Alten Reich und zum Territorialisierungsprozess im Herzogtum Württemberg an der Wende zur frühen Neuzeit. Leinfelden-Echterdingen 1998; Hans Martin Maurer: Der Arme Konrad als Schlüsselereignis württembergischer Geschichte. In: Jens-Uwe Wandel (Hg.): Der Arme Konrad. Schorndorf 1991, S. 8-25.

11 Vgl. dazu Brendle, Dynastie (wie Anm. 5). 
übergeben, ${ }^{12}$ der es von seinem Bruder Erzherzog Ferdinand verwalten ließ. ${ }^{13}$ Während der Zeit der habsburgischen Regierung zwischen 1520 und 1534 wurde die Ausbreitung der lutherischen Lehre entschieden bekämpft. ${ }^{14}$ So blieb bis zum Ende der habsburgischen Herrschaft das katholische Kirchenwesen weitgehend in Kraft, wenngleich die evangelische Bewegung in den benachbarten Reichsstädten auch ihre Strahlkraft auf das württembergische Territorium ausübte. Hier, in den Reichsstädten im Südwesten, konnte sich die Lehre Luthers als erstes entfalten, wobei die Städte immer mit einer gewissen Vorsicht bei der Durchführung der Reformation agierten, da sie von der Gegnerschaft des Kaisers, der ja auch ihr oberster Stadtherr war, wussten. ${ }^{15}$

Im Exil wandte sich der vertriebene Herzog der Reformation zu, wie er sie in der Schweiz kennenlernte. Politische wie auch religiöse Gründe spielten für diese Entscheidung eine Rolle. Herzog Ulrich war sich des politischen Vorteils eines Anschlusses an die Reformation wohl bewusst, seine Entscheidung diente ohne Zweifel der politischen Räson zur Rückeroberung seines Herzogtums. Denn in Landgraf Philipp von Hessen, an dessen Hof er sich seit 1526 aufhielt, dem politischen Haupt des deutschen Protestantismus, hatte er einen tatkräftigen Verbündeten gefunden, dessen dezidiertes Eintreten für das Evangelium sich mit einem scharfen Gegensatz zum Hause Habsburg verband. Der Reformation eine Bresche nach Oberdeutschland hinein zu schlagen - dies war das erklärte Ziel des hessischen Landgrafen gewesen, da seit dem Tod Zwinglis 1531 den evangelisch gewordenen Städten im Südwesten eine Anbindung fehlte. ${ }^{16}$ Nicht nur protestantische Reichsfürsten hatten die beiden Kriegsfürsten unterstützt, selbst die alten Feinde Ulrichs, die Herzöge von Bayern, waren neutral geblieben. $\mathrm{Zu}$ groß war der Unmut über die wachsende Macht Habsburgs, die sich auch in der Verdrängung einer angestammten Dynastie von ihren Herrschaftsrechten sowie der römischen Königswahl Ferdinands I. bemerkbar machte. Frankreich hatte

12 Zu Karl V. vgl. Alfred Kohler: Karl V. 1500-1558. Eine Biographie. München 1999; Luise Schorn-Schütte: Karl V. Kaiser zwischen Mittelalter und Neuzeit. München 2000; Alfred Kohler u. a. (Hg.): Karl V. 1500-1558. Neue Perspektiven seiner Herrschaft in Europa und Übersee. Wien 2002.

13 Zu Ferdinand I. vgl. Bernhard Sicken: Ferdinand I. (1556-1564). In: Anton Schindling, Walter Ziegler (Hg.): Die Kaiser der Neuzeit 1519-1918. Heiliges Römisches Reich, Österreich, Deutschland. München 1990, S. 55-77; Ernst Laubach: Ferdinand I. als Kaiser. Politik und Herrscherauffassung des Nachfolgers Karls V. Münster 2001; Martina Fuchs, Alfred Kohler (Hg.): Kaiser Ferdinand I. Aspekte eines Herrscherlebens. Münster 2003; Alfred Kohler: Ferdinand I. 1503-1564. Fürst, König und Kaiser. München 2003; Wilfried Seipel (Hg.): Kaiser Ferdinand I. 1503-1564. Das Werden der Habsburgermonarchie. Wien 2003.

14 Hans Puchta: Die Habsburgische Herrschaft in Württemberg 1520-1534. München 1967; Franz Brendle: Württemberg unter habsburgischer Herrschaft. In: Martina Fuchs, Alfred Kohler (Hg.): Kaiser Ferdinand I. Aspekte eines Herrscherlebens. Münster 2003, S. 177-190.

15 Volker Press: Die Reichsstadt in der altständischen Gesellschaft. In: Johannes Kunisch (Hg.): Neue Studien zur frühneuzeitlichen Reichsgeschichte. Berlin 1987, S. 9-42.

16 Vgl. dazu René Hauswirth: Landgraf Philipp von Hessen und Zwingli. Voraussetzungen und Geschichte der politischen Beziehungen zwischen Hessen, Konstanz, Ulrich von Württemberg und reformierten Eidgenossen 1526-1531. Tübingen/Basel 1968. 
ebenfalls eine willkommene Gelegenheit gesehen, dem großen Gegner auf europäischer Ebene eine empfindliche Niederlage beizubringen. ${ }^{17}$ So hatte König Franz I. eine bedeutende Geldsumme in München hinterlegt, mit der er das Vorhaben unterstützte.

Unter diesen Umständen hatten Herzog Ulrich von Württemberg und Landgraf Philipp von Hessen in einem raschen Kriegszug das Land zurückerobern können, zumal die habsburgischen Kräfte an der Türkenfront in Ungarn gebunden waren. ${ }^{18}$ Allerdings musste Herzog Ulrich 1534 im Vertrag von Kaaden zugestehen, sein Herzogtum nicht als ein unmittelbares Lehen vom Reich, sondern als Afterlehen von Österreich zu empfangen. Lange hatte sich der Herzog dagegen gewehrt, diese, für seine fürstliche Reputation schmähliche Regelung anzunehmen.

\section{Die Reformation des Landes}

Sogleich nach der Rückeroberung ging Herzog Ulrich an die reformatorische Umgestaltung seines Landes. ${ }^{19}$ Nach dem Vorbild des hessischen Landgrafen besaß die Reformation stark landesherrliche Züge, ${ }^{20}$ verschreckte zudem viele Zeitgenossen durch die rücksichtslose Säkularisation des Kirchenguts. ${ }^{21}$ Mit Erhard Schnepf aus Hessen berief er einen streng lutherisch orientierten Reformator, der in Württemberg seine Lebensaufgabe sah. ${ }^{22}$ Doch wollte Herzog Ulrich auch die zwinglianisch ausgerichtete Lehrmeinung angemessen berücksichti-

17 Karl Josef Seidel: Frankreich und die deutschen Protestanten. Die Bemühungen um eine religiöse Konkordie und die französische Bündnispolitik in den Jahren 1534/35. Münster 1970; Franz Brendle: Les relations franco-allemandes au temps de la Réforme. In: Les Annales de l'Académie d'Alsace 66 (2000), S. 17-32; Ders.: „Bündnis versus Bekenntnis“. Philipp der Großmütige von Hessen, die deutschen Protestanten und Frankreich im Zeitalter der Reformation. In: Historisches Jahrbuch 122 (2002), S. 87-109.

18 Jakob Wille: Philipp der Großmüthige von Hessen und die Restitution Ulrichs von Wirtemberg 1526-1535. Tübingen 1882; Thomas A. Brady: Princes’ Reformation Versus Urban Liberty: Strasbourg and the Restoration in Württemberg 1534. In: Ingrid Bátori (Hg.): Städtische Gesellschaft und Reformation. Stuttgart 1980, S. 265-291; Volker Press: Die württembergische Restitution von 1534 - reichspolitische Voraussetzungen und Konsequenzen. In: BWKG (1987), S. 44-71; Ders.: Ein Epochenjahr der Württembergischen Geschichte. Restitution und Reformation. In: ZWLG (1988), S. 203-234; Brendle, Dynastie (wie Anm. 5).

19 Zur württembergischen Reformation vgl. Martin Brecht, Hermann Ehmer: Südwestdeutsche Reformationsgeschichte. Zur Einführung der Reformation im Herzogtum Württemberg 1534. Stuttgart 1984; Brendle, Dynastie (wie Anm. 5); Hermann Ehmer: Die Reformation in Schwaben. Leinfelden-Echterdingen 2010.

20 Eike Wolgast: Formen landesfürstlicher Reformation in Deutschland. Kursachsen-Württemberg / Brandenburg-Kurpfalz. In: Leif Grane, Kai Hørby (Hg.): Die dänische Reformation vor ihrem internationalen Hintergrund. Göttingen 1990, S. 57-90.

21 Werner-Ulrich Deetjen: Studien zur Württembergischen Kirchenordnung Herzog Ulrichs 1534 1550. Das Herzogtum Württemberg im Zeitalter Herzog Ulrichs (1498-1550), die Neuordnung des Kirchengutes und der Klöster (1534-1547). Stuttgart 1981.

22 Zu Schnepf: Hermann Ehmer: Erhard Schnepf. Ein Lebensbild. In: BWKG 87 (1987), S. $72-$ 126; Wilhelm Schmidt: Der Weilburger Reformator Dr. Erhard Schnepf. In: Jahrbuch der hessischen 
gen, hatte die Reichsstadt Straßburg ihm doch wertvolle Unterstützung bei der Restitution seines Landes gewährleistet. Dem entsprach die Berufung des Konstanzer Predigers Ambrosius Blarer, der als Vertreter der oberdeutschen Lehrmeinung galt. ${ }^{23}$ Die oberdeutschen Theologen orientierten sich in ihrer Abendmahlslehre stärker an der Schweizer Reformation, weshalb sie oft von strengen Lutheranern skeptisch beurteilt wurden. Der Einflussbereich der beiden Reformatoren wurde durch die Weinsteige bei Stuttgart getrennt. Die visionäre Idee einer Vereinigung der konträren Lehrmeinungen stand sicher nicht hinter diesem Vorgehen - dafür war und blieb Ulrich viel zu sehr Pragmatiker.

Rasch kam es jedoch zu schweren Konflikten zwischen den beiden Prädikanten, die ihre Lehrstreitigkeiten offen auf der Kanzel austrugen und damit den Unmut auch des Herzogs auf sich zogen. Ulrichs Vermittlungsversuche konnten den Konflikt nur oberflächlich entschärfen. Dennoch trug der Herzog dazu bei, dass sich die oberdeutschen Städte zunehmend nach Wittenberg und nicht mehr nach Zürich orientierten und über die Stuttgarter Konkordie bis hin zur Wittenberger Konkordie 1536 schließlich den Anschluss an die Lehre Martin Luthers vollzogen. Mit seiner Kirchenordnung von 1536, dem Katechismus von Johannes Brenz, der Neuausrichtung der Tübinger Universität nach evangelischem Muster sowie der Kastenordnung, welche die unabhängige Existenz der Klöster beseitigte, beschritt der württembergische Herzog den Weg einer lutherischen Reformation seines Landes. Ambrosius Blarer hatte den Kampf verloren; enttäuscht verließ er seine Wirkungsstätte, die nun ganz im Sinne eines strengen Luthertums ausgerichtet wurde.

Doch ließen die Schwierigkeiten ebenfalls nicht auf sich warten. Dazu zählte in erster Linie das tiefgehende Zerwürfnis Herzog Ulrichs mit seinem Sohn Christoph, den er zunehmend als politischen Konkurrenten wahrnahm, weil er scheinbar im Sog der bayerischen Herzöge als „katholische Alternative“ zu seinem Vater in Württemberg installiert werden sollte. ${ }^{24}$ Christoph hatte bereits 1533 Ansprüche auf das Herzogtum Württemberg vor dem Schwäbischen Bund geltend gemacht. Er hatte sich ja im Gegensatz zu seinem Vater nichts zu Schulden kommen lassen. Da er in habsburgischer Obhut katholisch erzogen worden war, wäre damals eine Übergabe der Herrschaft an ihn auch für die Habsburger und die Bayernherzöge nicht ohne Reiz gewesen, rief allerdings den ungebremsten

kirchengeschichtlichen Vereinigung 47 (1996), S. 31-39; Hermann Ehmer: Erhard Schnepf und die Reformation in Württemberg. In: Hermle (wie Anm. 8), S. 255-288.

$23 \mathrm{Zu}$ Blarer: Martin Brecht: Ambrosius Blarers Wirksamkeit im Herzogtum Württemberg. In: Hermle (wie Anm. 8), S. 291-319; Hermann Ehmer: Ambrosius und Gerwig Blarer. Zwei Benediktiner in den Entscheidungsjahren der Reformation. In: BWKG 86 (1986), S. 196-214; Friedrich Held: Die Tätigkeit des Ambrosius Blarer im Herzogtum Württemberg in den Jahren 1534-1538. In: BWKG 65 (1965), S. 150-206. Bernd Moeller (Hg.): Der Konstanzer Reformator Ambrosius Blarer 1492-1564. Gedenkschrift zu seinem 400. Todestag. Konstanz/Stuttgart 1964; Bernd Möller: Zur Abendmahlstheologie Ambrosius Blarers. In: Max Geiger (Hg.): Gottesreich und Menschenreich, Ernst Staehelin zum 80. Geburtstag. Basel/Stuttgart 1969, S. 103-120.

24 Zum württembergischen Kronprinzenkonflikt vgl. Brendle, Dynastie (wie Anm. 5). 
Unwillen seines Vaters hervor. ${ }^{25}$ Zunächst war das Problem dadurch gelöst worden, dass Ulrich nach der Rückeroberung den Sohn in die Dienste des französischen Königs gab, um ihn von sich fernzuhalten. Am französischen Königshof konnte Christoph erste politische Erfahrungen sammeln und sich an das dortige Hofleben gewöhnen, was ihm jedoch die wiederholten Vorhaltungen der Stuttgarter Räte einbrachte, die sich um die Moral und den Charakter des jungen Herzogs sorgten. Brisanter allerdings entwickelte sich die politische Auseinandersetzung zwischen Vater und Sohn. Wilde Pläne über eine angebliche Herrschaftsübernahme Christophs entfremdeten ihn dem Vater vollends. Auf dem Höhepunkt dieses Kronprinzenkonflikts dachte Ulrich sogar an die Enterbung des ungeliebten Rivalen - erst die Vermittlung Landgraf Philipps und die Hinwendung Christophs zum evangelischen Glauben beendeten den Konflikt, der durchaus noch einmal das so mühsam Errungene wieder hätte in Frage stellen können. Im Vertrag von Reichenweier 1542 erhielt Christoph die linksrheinische Grafschaft Mömpelgard als Unterhalt zugesprochen, womit der Streit vorerst beigelegt und die dynastische Rangfolge gesichert war.

Nicht minder schwierig stellte sich die Lage nach dem verlorenen Schmalkaldischen Krieg für Herzog Ulrich dar. ${ }^{26}$ Nach dem Beitritt zu diesem evangelischen Verteidigungsbündnis hatte Ulrich treu seine Bundespflichten erfüllt und war gegen den anrückenden Kaiser Karl V. ins Feld gezogen, der 1546 die Religionsfrage im Reich mit militärischen Mitteln lösen wollte. Ulrich zählte zu den Besiegten und bekam den Zorn des kaiserlichen Siegers zu spüren. Spanische Truppen wurden ins Land gelegt und ihm selbst eine schmähliche Unterwerfung abverlangt. Jetzt bekam der Herzog die Afterlehensschaft Württembergs in ihrer vollen Tragweite zu spüren. Denn König Ferdinand strengte einen Felonieprozess wegen Verletzung der Treuepflicht gegen den württembergischen Herzog an, dem damit erneut der Verlust des Landes drohte. Spanische Truppen setzten das kaiserliche Interim mit Gewalt im Territorium durch, eine Zwischenlösung in der strittigen Religionsfrage, die eine Rückkehr der Protestanten zur alten Kirche bei Gewährung des Laienkelchs und der Priesterehe vorsah. ${ }^{27}$ Zahlreiche evangelische Prediger waren gezwungen, das Land zu verlassen, Ulrich stand vor dem Ruin seiner gewagten Politik. ${ }^{28}$ Nur der Tod rettete ihn 1550 vor dem Einzug seiner Herrschaft, die nun an seinen Sohn Herzog Christoph überging,

25 Joachim Lauchs: Bayern und die deutschen Protestanten 1534-1546. Deutsche Fürstenpolitik zwischen Konfession und Libertät. Neustadt a. d. Aisch 1978.

26 Zum Schmalkaldischen Krieg vgl. Gabriele Haug-Moritz: Der Schmalkaldische Krieg (1546/47) - ein kaiserlicher Religionskrieg? In: Franz Brendle, Anton Schindling (Hg.): Religionskriege im Alten Reich und in Alteuropa. Münster ${ }^{2} 2010$, S. 93-105; Horst Rabe: Abschied vom Ketzerrecht? Zur Religionspolitik Karls V. In: Irene Dingel u.a. (Hg.): Reformation und Recht, Festgabe für Gottfried Seebaß zum 65. Geburtstag. Gütersloh 2002, S. 40-57.

27 Zum Interim vgl. die beiden Sammelbände Luise Schorn-Schütte (Hg.): Das Interim 1548/50. Herrschaftskrise und Glaubenskonflikt. Heidelberg 2005; Irene Dingel, Günter Wartenberg (Hg.): Politik und Bekenntnis. Die Reaktionen auf das Interim von 1548. Leipzig 2006.

28 Zum Interim in Württemberg vgl. Brendle, Dynastie (wie Anm. 5); Gustav Bossert: Das Interim 
der bislang in Mömpelgard erste Regierungserfahrungen gesammelt hatte. Erst der Passauer Vertrag $1552^{29}$ und der Augsburger Religionsfriede 1555 beendeten die unsichere Lage nicht nur im Südwesten, sondern auch im übrigen Deutschland. ${ }^{30}$ Den weltlichen Reichsständen war fortan freigestellt, die Reformation einzuführen. Dabei bestimmte der Landesherr die Konfession seiner Untertanen: „cuius regio, eius religio“.

\section{Der Organisator von Staat und Kirche: Christoph von Württemberg}

Nach dem Augsburger Religionsfrieden sollte Württemberg bald eine dominierende Rolle im deutschen Protestantismus einnehmen. Der schnell einsetzende Erfolg in der Landes- und Reichspolitik Herzog Christophs ist auf mehrere Ursachen zurückzuführen. ${ }^{31}$ Zunächst einmal musste er nicht mit den Vorbehalten kämpfen, die seinem umtriebigen, schwierigen Vater zeitlebens entgegengebracht wurden. Zudem kamen ihm zu Beginn seiner Regentschaft die reichspolitischen Veränderungen zu Hilfe, da mit dem Fürstenkrieg von 1552 die dominante Rolle Kaiser Karls V. und des habsburgischen Hauses im Reich gebrochen worden war und die ständischen Kräfte gestärkt wurden. ${ }^{32}$ Interim und Felonieprozess waren abgetan, das landesfürstliche Reformationsrecht an ihre Stelle getreten. Zwar hatte Christoph aus Rücksicht auf Karl V. als einer der wenigen protestantischen Reichsfürsten seine Gesandten noch zum Konzil nach Trient geschickt, - wenngleich er zuvor 1551 mit der Confessio Virtembergica sein

in Württemberg. Halle 1895; Armin Kohnle: Die Folgen des Interims am Beispiel Württembergs: In: Dingel, Wartenberg (wie Anm. 27), S. 83-96.

29 Volker Henning Drecoll: Der Passauer Vertrag (1552). Einleitung und Edition. Berlin/New York 2000; Winfried Becker (Hg.): Der Passauer Vertrag von 1552. Politische Entstehung, reichsrechtliche Bedeutung und konfessionsgeschichtliche Bewertung. Neustadt a. d. Aisch 2003.

30 Zum Augsburger Religionsfrieden und seinen Auswirkungen vgl. Hermann Tüchle: Der Augsburger Religionsfriede. Neue Ordnung oder Kampfpause. In: Zeitschrift des historischen Vereins für Schwaben 61 (1955), S. 323-340; Gerhard Pfeiffer: Augsburger Religionsfriede. In: TRE 4 (1979), S. 639-645; Axel Gotthard: Der Augsburger Religionsfrieden. Münster 2004; Carl A. Hoffmann u. a. (Hgg.): Als Frieden möglich war. 450 Jahre Augsburger Religionsfrieden. Begleitband zur Ausstellung im Maximilianmuseum Augsburg. Regensburg 2005; Andreas Schmauder (Hg.): Hahn und Kreuz. 450 Jahre Parität in Ravensburg. Konstanz 2005; Wolfgang Wüst (Hg.): Der Augsburger Religionsfriede 1555. Ein Epochenereignis und seine regionale Verankerung. Augsburg 2005; Heinz Schilling, Heribert Smolinsky (Hg.): Der Augsburger Religionsfrieden 1555. Münster 2007.

31 Zur Person Herzog Christophs vgl. Hans-Martin Maurer: Herzog Christoph (1550-1568). In: Uhland (wie Anm. 8), S. 136-162; Franz Brendle, Christoph. In: Lorenz, Das Haus Württemberg (wie Anm. 8), S. 108-111; Brendle, Dynastie (wie Anm. 5); Eberhard Fritz: Herzog Christoph von Württemberg. In: Hermle (wie Anm. 8), S. 199-225; Matthias Langensteiner: Für Land und Luthertum. Die Politik Herzog Christophs von Württemberg (1550-1568). Köln 2008.

32 Zum Fürstenkrieg vgl. Franz Brendle: Um Erhalt und Ausbreitung des Evangeliums: Die Reformationskriege der deutschen Protestanten. In: Franz Brendle, Anton Schindling (Hg.): Religionskriege im Alten Reich und in Alteuropa. Münster ${ }^{2} 2010$, S. 71-92. 
Bekenntnis und das seines Landes eindeutig formulieren ließ.$^{33}$ Johannes Brenz, der ehemalige Reformator von Schwäbisch Hall, der nach Württemberg gerufen worden war, hatte das württembergische Bekenntnis verfasst - er sollte der wichtigste theologische Berater des Herzogs werden. ${ }^{34}$ Denn seit 1555 ermöglichten Herzog Christoph die Regelungen des Religionsfriedens den zentralen und systematischen Aufbau eines lutherischen Landesstaats. Bei all seiner Bekenntnistreue korrespondierte damit auf der Reichsebene eine kluge Rücksichtnahme auf den Kaiser, die im Hinblick auf die Afterlehensschaft durchaus geboten war. Dies äußerte sich unter anderem darin, dass er mit anderen Reichsfürsten unterschiedlicher konfessioneller Ausrichtung (Kursachsen, Hessen, Bayern) eine Art Mittelpartei im Reich bildete, welche die Errungenschaften des mühsam erkämpften Augsburger Religionsfriedens unter allen Umständen bewahren wollte. ${ }^{35}$ In zahlreichen Religionsgesprächen kämpften württembergische Theologen im Auftrag ihres Herrn um die Einheit des deutschen Protestantismus, wenngleich die unterschiedlichen Lager kaum zusammenzuführen waren.

Entschieden gestaltete sich allerdings sein Auftreten gegen den erstarkenden Calvinismus, der vor allem seit dem Übertritt der Kurpfalz 1563 im Reich an Boden gewann.$^{36}$ So versuchte der württembergische Herzog auf dem Augsburger Reichstag 1566, die Reformierten aus dem Augsburger Religionsfrieden zu drängen, scheiterte mit seiner unnachgiebigen Haltung allerdings an der Irenik des sächsischen Kurfürsten. ${ }^{37}$ Ein aktives Eintreten für die calvinistischen Huge-

33 Ernst Bizer (Hg.): Confessio Virtembergica. Das württembergische Bekenntnis von 1551. Stuttgart 1952; Martin Brecht, Hermann Ehmer (Hg.): Confessio Virtembergica. Das Württembergische Bekenntnis von 1552. Holzgerlingen 1999.

$34 \mathrm{Zu}$ Johannes Brenz vgl. Martin Brecht: Johannes Brenz. Neugestalter von Kirche, Staat und Gesellschaft. Stuttgart 1971; Hans-Martin Maurer, Kuno Ulshöfer: Johannes Brenz und die Reformation in Württemberg. Stuttgart/Aalen 1974; Martin Brecht: Brenz, Johannes. In: TRE 7 (1981), S. 170 181; Ders.: Johannes Brenz. In: Martin Greschat (Hg.): Gestalten der Kirchengeschichte, Bd. 6. Stuttgart 1981, S. 103-117; Isabella Fehle (Hg.): Johannes Brenz 1499-1570. Prediger - Reformator Politiker. Begleitbuch zur Ausstellung in Schwäbisch Hall und Stuttgart 1999. Schwäbisch Hall 1999; Hermann Ehmer: Johannes Brenz - Ein Lebensbild zum 500. Geburtstag. In: Schwäbische Heimat 49 (1999), S. 56-65, 156-163; Martin Brecht: Johannes Brenz: Stiftspropst, Prediger, Reformator Württembergs und Rat Herzog Christophs. In: Hermle (wie Anm. 8), S. 321-344.

35 Vgl. dazu auch Bernhard Sicken: Der Heidelberger Verein (1553-1556). Zugleich ein Beitrag zur Reichspolitik Herzog Christophs von Württemberg in den ersten Jahren seiner Regierung. In: ZWLG 32 (1973), S. 320-435.

36 Vgl. dazu Volker Press: Calvinismus und Territorialstaat. Regierung und Zentralbehörden der Kurpfalz 1559 bis 1619. Stuttgart 1970; Ders.: Außerhalb des Religionsfriedens? Das reformierte Bekenntnis im Reich bis 1648. In: Günter Vogler (Hg.): Wegscheiden der Reformation. Weimar 1994, S. 309-335; Eike Wolgast: Reformierte Konfession und Politik im 16. Jahrhundert. Studien zur Geschichte der Kurpfalz im Reformationszeitalter. Heidelberg 1998.

37 Walter Hollweg: Der Augsburger Reichstag von 1566 und seine Bedeutung für die Entstehung der Reformierten Kirche und ihres Bekenntnisses. Neukirchen-Vluyn 1964; Maximilian Lanzinner (Bearb.): Der Reichstag zu Augsburg 1566. München 2002; Zur Religionspolitik Maximilians II. vgl. Manfred Rudersdorf: Maximilian II. (1564-1576). In: Schindling, Ziegler (wie Anm. 13), S. 79-97; Friedrich Edelmayer, Alfred Kohler (Hg.): Kaiser Maximilian II. Kultur und Politik im 16. Jahrhundert. Wien/München 1992; Maximilian Lanzinner: Friedenssicherung und politische 
notten in Frankreich war demnach von Christoph auch nicht zu erwarten. Doch setzte er sich immer wieder für Glaubensflüchtlinge aus Frankreich ebenso ein wie für die Protestanten, die den Verfolgungen in den habsburgischen Erblanden zu entkommen suchten. Slowenische Theologen wie Primus Truber ${ }^{38}$ und Pier Paolo Vergerio ${ }^{39}$ fanden in Württemberg Aufnahme und konnten von hier aus in ihrem Heimatland reformatorisch wirken. Der in Urach und Tübingen wirkende Drucker Hans Ungnad von Sonnegg verbreitete ihre Bibelübersetzung und ihre Katechismen und schuf damit die Grundlage für die Entstehung der modernen slowenischen Schriftsprache. ${ }^{40}$ So wirkte das württembergische Herzogtum weit in das evangelische Europa hinaus.

Zahlreiche Heiratsprojekte mit anderen protestantischen Territorien, vor allem Hessen, sicherten die württembergische Position innerhalb des evangelischen Deutschland ab. Herzog Christoph von Württemberg wuchs immer stärker in die politische Führungsrolle des deutschen Protestantismus hinein, die der alternde Landgraf Philipp von Hessen nicht mehr ausfüllen konnte. Gestützt auf seine politischen und dynastischen Verbindungen und gestärkt durch seine persönliche Autorität konnte Herzog Christoph so auch eine zentrale Rolle in der Reichspolitik dieser Jahre spielen, die noch gestärkt wurde durch sein Engagement als Hauptmann des Schwäbischen Reichskreises. ${ }^{41}$ Hier trat er entschlossen für die württembergischen Interessen ein, wenn er etwa 1552/53 sogar militärisch gegen die Pläne Kaiser Karls V. vorging, der mit der Vereinigung des Deutschor-

Einheit des Reiches unter Kaiser Maximilian II. (1564-1576). Göttingen 1993; Andreas Edel: Der Kaiser und Kurpfalz. Eine Studie zu den Grundelementen politischen Handelns bei Maximilian II. (1564-1576). Göttingen 1997.

38 Theodor Elze: Primus Trubers Briefe. Tübingen 1897; Mirko Rupel: Primus Truber. Leben und Werk des slowenischen Reformators. München 1965; Christoph Weismann: Die slowenische Kirchenordnung Primus Trubers von 1564. Ein Beitrag zur Bibliographie der südslawischen Reformationsdrucke. In: Gutenberg-Jahrbuch 1972, S. 197-210; Rolf-Dieter Kluge (Hg.): Ein Leben zwischen Laibach und Tübingen. Primus Truber und seine Zeit. Intentionen, Verlauf und Folgen der Reformation in Württemberg und Innerösterreich. München 1995; Franz Brendle: Vom Leben und Sterben Herr Primus Trubers, in: Lorenz, Primus Truber (wie Anm. 1), S. 13-22.

39 Eduard von Kausler, Theodor Schott (Hg.): Briefwechsel zwischen Christoph, Herzog von Württemberg, und Petrus Paulus Vergerius. Stuttgart 1875; Gerhard Müller: Pietro Paolo Vergerio in päpstlichen Diensten 1532-1536. In: Zeitschrift für Kirchengeschichte 77 (1966), S. 341-348.

40 Christian Friedrich Schnurrer: Slavischer Bücherdruck in Württemberg im 16. Jahrhundert. Ein literarischer Bericht. Tübingen 1799; Bernhard H. Zimmermann: Hans Ungnad, Freiherr von Sonneck, als Förderer reformatorischer Bestrebungen bei den Südslawen. In: Südostdeutsche Forschungen 2 (1937), S. 36-58; Ernst Benz: Hans von Ungnad und die Reformation unter den Südslaven. In: Zeitschrift für Kirchengeschichte 58 (1939), S. 387-475; Günther Stökl: Der Beginn des Reformationsschrifttums in slowenischer Sprache. In: Südostforschungen 15 (1956), S. 268-277; Klaus Schreiner: Die Uracher Druckerei Hans Ungnads - ein Opfer der Gegenreformation? In: Gutenberg-Jahrbuch 1972, S. 217-236; Rolf Vorndran: Kurzer Überblick über die Drucke der südslawischen Bibelanstalt Urach. In: Gutenberg-Jahrbuch 1976, S. 291-297; Christoph Weismann: „Der Winden, Crabaten und Türken Bekehrung“. Reformation und Buchdruck bei den Südslawen 1550-1595. In: Kirche im Osten 29 (1986), S. 9-37; Hermann Ehmer: Der slawische Buchdruck des Hans Ungnad in Urach. In: Kluge, Ein Leben (wie Anm. 38), S. 438-451.

41 Langensteiner (wie Anm. 31). 
densterritoriums um Mergentheim und der Fürstpropstei Ellwangen einen katholischen Sperrriegel am Rande Württembergs errichten wollte. Ein Kriegszug Christophs beendete die Herrschaft des Deutschmeisters Wolfgang Schutzbar, genannt Milchling, in Ellwangen. ${ }^{42}$ Eine enge politische Freundschaft verband ihn am Ende seiner Regierung mit Kaiser Maximilian II., dessen Sympathien für den Protestantismus offenkundig waren, wenngleich sich weitergehende Hoffnungen auf einen Bekenntniswechsel des Kaisers nicht erfüllten. Dennoch: Rücksichtnahme auf Kaiser und Reich waren die Voraussetzung für Christophs erfolgreiche Landespolitik.

Auf diesem Gebiet liegen ohne Zweifel die nachhaltigsten Verdienste Herzog Christophs. Denn seine Organisation und Struktur des württembergischen Landesstaats wurden zum Vorbild für das lutherische Deutschland. ${ }^{43}$ Mit dem Stuttgarter Stiftspropst Johannes Brenz hatte er einen kompetenten Helfer an seiner Seite, der maßgeblich für die Große württembergische Kirchenordnung von 1559 die Verantwortung trug. ${ }^{44}$ Der Kirchenrat wurde als oberste kirchliche Zentralbehörde eingerichtet und beaufsichtigte nicht nur Predigt und Seelsorge, sondern auch Wirtschaft und Finanzen. Über die Superintendenten, welche die Stellung eines Bischofs innehatten, wurde der Kontakt vom Kirchenrat zu den einzelnen Gemeinden und Pfarrern sichergestellt. ${ }^{45}$ Das eingezogene Kirchengut wurde der landesherrlichen Verwaltung unterstellt und das klösterliche Leben auf die Grundlage des evangelischen Bekenntnisses gestellt. Es wurden 13 Klosterschulen (Bebenhausen, Maulbronn, Denkendorf, Blaubeuren u. a.) mit humanistischen Bildungsidealen eingerichtet, die zur Ausbildung des theologischen Nachwuchses dienen sollten. ${ }^{46}$ Als Prälaten waren die Vorsteher dieser evangelischen Schulen auf dem Landtag vertreten. Sozial rekrutierten sie sich ebenso wie die herzoglichen Beamten aus der bürgerlichen Schicht der Ehrbarkeit, die damit zur bestimmenden Kraft des frühneuzeitlichen Landesstaates avancierte. ${ }^{47}$ Auch

42 Vgl. dazu Franz Brendle: Das habsburgische Kaisertum und die Fürstpropstei Ellwangen in der Frühen Neuzeit. In: ZWLG 76 (2017), S. 75-94.

43 Hans-Martin Maurer: Herzog Christoph als Landesherr. In: BWKG 68/69 (1968/69), S. 112 138.

44 Julius Rauscher: Zur Entstehung der großen württembergischen Kirchenordnung des Jahres 1559. In: Festschrift Karl Müller. Tübingen 1922, S. 171-177; Sabine Arend u. a. (Hg.): Die württembergische Kirchenordnung von 1559 im Spannungsfeld von Religion, Politik und Gesellschaft. Epfendorf 2013.

45 Martin Brecht: Kirchenordnung und Kirchenzucht in Württemberg vom 16. bis zum 18. Jahrhundert. Stuttgart 1967.

46 Gustav Lang: Geschichte der württembergischen Klosterschulen von ihrer Stiftung bis zu ihrer endgültigen Verwandlung in Evangelisch-theologische Seminare. Stuttgart 1938; Hermann Ehmer: Valentin Vannius und die Reformation in Württemberg. Stuttgart 1976; Ders.: Der Humanismus an den evangelischen Klosterschulen in Württemberg. In: Wolfgang Reinhard (Hg.): Humanismus im Bildungswesen des 15. und 16. Jahrhunderts. Weinheim 1984, S. 121-133.

47 Julian Kümmerle: Luthertum, humanistische Bildung und württembergischer Territorialstaat. Die Gelehrtenfamilie Bidembach vom 16. bis zum 18. Jahrhundert. Stuttgart 2008; Gabriele Haug-Moritz: Die württembergische Ehrbarkeit. Annäherungen an eine bürgerliche Machtelite der Frühen Neuzeit. Ostfildern 2009. 
im Bereich der Volksschulbildung wurden neue Wege beschritten, um eine flächendeckende Ausbildung selbst in den Dörfern zu gewährleisten. ${ }^{48}$ Analog wurde eine ähnlich konstruierte Kirchenordnung für die linksrheinischen Gebiete des Herzogtums in Mömpelgard, Horburg und Reichenweier erlassen. ${ }^{49}$ Nach der Ausbildung in den württembergischen Klosterschulen ${ }^{50}$ sollten die zukünftigen Theologen mit einem Stipendium des Landesherrn am Tübinger Stift studieren. ${ }^{51}$ So war auch die Tübinger Universität fest im herzoglichen Schulund Bildungssystem verankert. ${ }^{52}$ Die theologischen Professuren waren eng mit den kirchlichen Ämtern an der Tübinger Stiftskirche verbunden. ${ }^{53}$ Auch besaß die theologische Fakultät Tübingens eine Ausstrahlungskraft weit über das Land hinaus. Württembergische Theologen wirkten an vielen Stellen in benachbarten Territorien und Städten und begründeten damit den Ruf Württembergs als eines lutherischen Musterstaates. ${ }^{54}$ Mit Jakob Andreae, Jakob Heerbrand ${ }^{55}$, Dietrich Schnepf und Johannes Brenz hatte in Tübingen eine Professorengruppe zusammengefunden, welche an der Spitze ihrer Zunft stand und nicht nur das Tübinger Luthertum, sondern das evangelische Deutschland prägen sollte. Dass Württemberg das „lutherische Spanien“ genannt wurde, war daher in keiner Weise abschätzig gemeint, sondern spiegelte die Führungsrolle wider, die Herzog Christoph, seine Theologen und Räte für die Ausbildung der lutherischen Orthodoxie spielten.

48 Eugen Schmid: Geschichte des Volksschulwesens in Altwürttemberg. Stuttgart 1927.

49 Franz Brendle: Die „Einführung“ der Reformation in Mömpelgard, Horburg und Reichenweier zwischen Landesherrn, Theologen und Untertanen. In: Sönke Lorenz, Peter Rückert (Hg.): Württemberg und Mömpelgard. 600 Jahre Begegnung. Leinfelden-Echterdingen 1999, S. 145-168; Ders.: Les enclaves territoriales et confessionelles du duché de Wurtemberg: Montbéliard, Horbourg et Riquewihr. In: Paul Delsalle, André Ferrer (Hg.): Les enclaves territoriales aux Temps Modernes (XIVe-XVIIIe siècles). Colloque international de Besançon 4 et 5 octobre 1999. Besançon 2000. S. 419-430; Ders.: Schwaben - Elsass - Mömpelgard: politische und theologische Wechselwirkungen im Reformationsprozess. In: Ulrich A. Wien, Volker Leppin (Hg.): Kirche und Politik am Oberrhein im 16. Jahrhundert. Reformation und Macht im Südwesten des Reiches. Tübingen 2015, S. 97-110. 50 Vgl. dazu: Hermann Ehmer: Der Humanismus an den evangelischen Klosterschulen in Württemberg. In: Wolfgang Reinhard (Hg.): Humanismus im Bildungswesen des 15. und 16. Jahrhunderts. Weinheim 1984, S. 121-133; Elizabeth Lewis Pardoe: Education, Economics, and Orthodoxy: Lutheran Schools in Württemberg, 1556-1617. In: Archiv für Reformationsgeschichte 91 (2000), S. 285-315.

51 Martin Leube: Die Geschichte des Tübinger Stifts im 16. und 17. Jahrhundert. Stuttgart 1921; Joachim Hahn/Hans Mayer: Das Evangelische Stift in Tübingen. Geschichte und Gegenwart - zwischen Weltgeist und Frömmigkeit. Stuttgart 1985.

52 Franz Brendle: Tübingen, Universität. In: LThK 10 (2001), Sp. 285-287.

53 Martin Brecht (Hg.): Theologen und Theologie an der Universität Tübingen. Beiträge zur Geschichte der Evangelisch-Theologischen Fakultät. Tübingen 1977.

54 Martin Brecht: Herkunft und Ausbildung der protestantischen Geistlichen des Herzogtums Württemberg im 16. Jahrhundert. In: Zeitschrift für Kirchengeschichte 80 (1969), S. 163-175.

$55 \mathrm{Zu}$ Heerbrand: Volker Leppin: Jakob Heerbrand. Ein Superattendent in den Diskursen seiner Zeit. In: Volker Henning Drecoll (Hg.): 750 Jahre Augustinerkloster und Evangelisches Stift in Tübingen. Tübingen 2018, S. 83-109; Siegfried Raeder: Jakob Heerbrand. In: Friedrich Hertel (Hg.): In Wahrheit und Freiheit. 450 Jahre Evangelisches Stift in Tübingen. Stuttgart 1986, S. 81-98. 


\section{Die europäische Dimension der württembergischen Reformation}

In der Regierungszeit Herzog Christophs von Württemberg waren zahlreiche Glaubensflüchtlinge aus Österreich, Bayern und Tirol nach Württemberg gekommen. Neben Primus Truber hatte auch Pier Paolo Vergerio, ehemals päpstlicher Nuntius, dann Bischof von Capo d'Istria, nach seiner Hinwendung zur Reformation 1553 Aufnahme gefunden und diente den württembergischen Herzögen als Diplomat in religionspolitischen Angelegenheiten unter anderem in Graubünden und Polen. Hans Freiherr Ungnad von Sonnegg gründete in Urach eine slawische Druckerei und unterstützte damit das Anliegen der Krainer Reformation. Denn er war derjenige, der die von Primus Truber ins Slowenische übersetzten Schriften zum Druck brachte. Truber hatte dazu zuerst eine slowenische Schriftsprache entwickeln müssen. Von Württemberg aus trieben die slowenischen Glaubensflüchtlinge dabei die Reformation in ihrem Heimatland voran. Die enge Verbindung zwischen der Tübinger Universität und dem innerösterreichischen Exulantenkreis um Primus Truber ist auch im Zusammenhang mit der konfessionspolitischen Ausrichtung der Tübinger Theologen zu sehen, deren Aktivitäten in den 1580er und 1590er Jahren nach Südosteuropa wiesen und sich bis nach Konstantinopel erstreckten. ${ }^{56}$ Unter der Regierung des Patriarchen Jeremias II. begannen die Beziehungen zwischen Griechen und deutschen Protestanten, die eine Vereinigung der Kirchen erstrebten. Ihre Hauptvertreter waren in Tübingen Jakob Andreae ${ }^{57}$ und Martin Crusius. ${ }^{58}$ Die Protestanten sahen eine Möglichkeit des gemeinsamen Vorgehens im Kampf gegen die katholische Kir-

56 Vgl. dazu Dorothea Wendebourg: Reformation und Orthodoxie. Der ökumenische Briefwechsel zwischen der Leitung der württembergischen Kirche und Patriarch Jeremias II. von Konstantinopel in den Jahren 1573-1581. Göttingen 1986; George Elias Zachariades: Tübingen und Konstantinopel. Martin Crusius und seine Verhandlungen mit der griechisch-orthodoxen Kirche. Göttingen 1941.

$57 \mathrm{Zu}$ Andreae vgl. Heinrich Gürsching: Jakob Andreae und seine Zeit. In: BWKG 54 (1954), S. 132-156; Rosemarie Müller-Streisand: Theologie und Kirchenpolitik bei Jakob Andreä bis zum Jahr 1568. In: Ebda. 60/61 (1960/61), S. 224-395; Martin Brecht: Andreae, Jakob (1528-1590). In: TRE 2 (1978), S. 672-680; Jobst Christian Ebel: Jacob Andreae (1528-1590) als Verfasser der Konkordienformel. In: Zeitschrift für Kirchengeschichte 89 (1978), S. 78-119; Siegfried Raeder: Jakob Andreae. Ein Leben für Reformation und Eintracht im Glauben. In: Theologische Beiträge 21 (1990), S. 244-263; Hermann Ehmer (Hg.): Leben des Jakob Andreae, Doktor der Theologie, von ihm selbst mit großer Treue und Aufrichtigkeit beschrieben, bis auf das Jahr 1562. Lateinisch und Deutsch. Stuttgart 1991; Siegfried Raeder: Jakob Andreae und die Reformation in Wiesensteig, Öttingen und Wachendorf. In: Hermle (wie Anm. 8), S. 365-394; Ulrike Ludwig: Philippismus und orthodoxes Luthertum an der Universität Wittenberg. Die Rolle Jakob Andreäs im lutherischen Konfessionalisierungsprozeß Kursachsens (1576-1589). Münster 2009; Christoph Weismann: Auf Kanzeln, Kathedern und in Kutschen. Jakob Andreae als Universitäts- und Kirchenpolitiker. In: Ulrich $\operatorname{Köpf}(\mathrm{Hg}$.): Die Universität Tübingen zwischen Reformation und Dreißigjährigem Krieg. Festgabe für Dieter Mertens zum 70. Geburtstag. Ostfildern 2010, S. 119-140.

58 Franz Brendle: Martin Crusius. Humanistische Bildung, schwäbisches Luthertum und Griechenlandbegeisterung. In: Franz Brendle u. a. (Hgg.): Deutsche Landesgeschichtsschreibung im Zeichen des Humanismus. Stuttgart 2001, S. 145-163; Hubert Cancik: Crusius contra Frischlinum. Geschichte einer Feindschaft. In: Sabine Holtz, Dieter Mertens (Hg.): Nicodemus Frischlin (15471590). Poetische und prosaische Praxis unter den Vorbedingungen des konfessionellen Zeitalters. 
che. Die Protestanten wollten beweisen, dass die Lehre der neuen Kirche mit derjenigen der griechischen Kirche kongruent sei. Bekanntlich haben diese Versuche nicht zum Erfolg geführt. Der Patriarch erklärte, dass die Kirche des Ostens die einzige sei, die seit den frühen Zeiten die Wahrheit besitze und die echte Lehre der frühchristlichen Kirche ohne jegliche Änderung in Auffassungen, Gedanken oder Glaubenslehren bewahrt habe. Er begründete diese Überzeugung allein mit dem Hinweis auf die Heilige Schrift, die Entscheidungen der ökumenischen Konzilien und die Werke der griechischen Väter (Basilius, Johannes Chrysostomus). Die Lehre und den Glauben der Protestanten zog er überhaupt nicht in Betracht. Die Einigung war nach Auffassung der Autoritäten in Konstantinopel nur unter einer Bedingung möglich: vollständige Annahme der Lehren, Glaubenssätze und Dogmen der griechischen Kirche.

Auch die kurzzeitige Reformationsbewegung im Fürstentum Moldau anfangs der 1560er Jahre wurde in Tübingen mit Interesse registriert. Initiator und Träger dieses Unternehmens war ein gebürtiger Grieche, Jakobus Heraklides Despota, der von den Türken vertrieben worden war. ${ }^{59}$ Bei einem Aufenthalt in Wittenberg, wo er die Reformation kennen gelernt hatte, begab er sich auf abenteuerliche Reisen quer durch Europa. Nachdem sich seine Hoffnungen auf eine Wiedergewinnung seiner Herrschaft über die griechischen Inseln zerschlagen hatten, dachte er daran, sich in den östlichen Provinzen, die in den Grenzkriegen mit den Türken so oft ihren Herrscher wechselten, ein Reich zu erobern. Kurzfristig gelang ihm dieses Vorhaben im Fürstentum Moldau, in dem er 1561 mit der Reformation des Landes begann. Er wollte in der Moldau ein Bollwerk eines evangelischen Staates aufrichten, von dem aus die türkische Herrschaft erfolgreich zurückgedrängt werden sollte. Allerdings war die außenpolitische Lage so, dass er zunächst einmal eine Verständigung mit dem Sultan suchen musste, um seine labile Herrschaft vorläufig zu sichern und auszubauen. So unterstellte er sich dem Sultan und nahm von ihm, nach Zahlung einer Tributsumme, die türkische Vasallenschaft an. Das Land hat allerdings den raschen Anschluss an den Westen und an die Reformation nicht so ohne weiteres ertragen. Die Religion des Landes war die östlich-orthodoxe. So kam die Herrschaft des Herakliden nach nur zwei Jahren 1563 schon wieder zum Ende.

Das Reformationswerk in der Moldau erregte auch die Aufmerksamkeit von Primus Truber und Hans Ungnad. Sie erblickten in der vom Herakliden für die Reformation erschlossenen Moldau sogleich ein Absatzgebiet für die kyrillischen Drucke und schickten ihm einen Gesandten, Wolfgang Schreiber, der Heraklides die bisher erschienenen slawischen Drucke überreichte und sich erbot, eine Druckerei für Übersetzungen reformatorischer Schriften in die walachische

Stuttgart-Bad Cannstatt 1999, S. 261-295; Reinhold Stahlecker: Martin Crusius und Nicodemus Frischlin. In: ZWLG 7 (1943), S. 323-366.

59 Vgl. dazu Ernst Benz: Wittenberg und Byzanz. Zur Begegnung und Auseinandersetzung der Reformation und der östlich-orthodoxen Kirche. Marburg 1949, hier S. 141-246. 
Sprache einzurichten. Doch ging der Gesandte über den Auftrag seiner Auftraggeber hinaus, indem er die Unterstellung des Landes unter die Herrschaft des Kaisers forderte und dafür nach Konstantinopel ausgeliefert wurde, wo er mehrere Jahre bis 1565 als Gefangener verbrachte. Auf der anderen Seite hatte Truber zwei uskokische Priester griechischen Glaubens nach Urach gebracht, um ihm bei seiner Übersetzungsarbeit zu helfen. ${ }^{60}$ Allerdings bereiteten die beiden Uskoken in Urach viel Ärger, weil sie ihre Tracht als griechische Mönche beibehielten und zum großen Erstaunen der Uracher Bürger in ihren schwarzen Kutten und ihrer hohen Kamilowka mit langen Haaren und Bärten herumliefen. Zudem lehnten sie die schwäbische Küche entschieden ab, aßen kein Fleisch und taten sich durch exzessiven Alkoholkonsum hervor. ${ }^{61}$

Über die Verhältnisse in Südosteuropa wusste man bereits vorher in Tübingen aus erster Hand Bescheid. Jakob Andreae war es gewesen, der Truber dabei unterstützte, dass dieser 1567 noch einmal insgeheim nach Laibach reisen konnte. Trubers Bericht über die Verhältnisse in seiner Heimat benutzte Andreae für seine „Dreizehn Predigten vom Türken“, in denen er auf die doppelte Gefahr seiner evangelischen Glaubensgenossen in Slowenien aufmerksam machte: zwischen päpstlicher Nachstellung einerseits und türkischer Verfolgung andererseits aufgerieben zu werden. ${ }^{62}$ Der enge Austausch zwischen der Krain und Württemberg wurde in den letzten Jahren Trubers in Derendingen intensiviert und ausgebaut. Truber vermittelte Andreas Spindler, der das Tübinger Stift absolviert hatte, als Superintendenten nach Laibach. Selbst der in Tübingen in Ungnade gefallene Nikodemus Frischlin konnte 1582 durch die Unterstützung Trubers die Stelle des Rektors der Laibacher Landschaftsschule antreten, die von Truber ins Leben gerufen worden war. Dass Jakob Andreae ihn dabei unterstützte, hat Truber nicht vergessen. So setzte er sich für die Unterzeichnung der Konkordienformel durch die Landstände von Krain, Kärnten und Steiermark ein.

Aber auch umgekehrt kamen zahlreiche Studenten aus der Krain nach Tübingen. Grundlage dafür war das Testament von Michael Tiffernus, des slowenischen Erziehers und Freunds Herzog Christophs. ${ }^{63}$ Dieses Testament zeigt nicht nur seine enge Verbundenheit zu Herzog Christoph, sondern auch sein Engagement für das Herzogtum und die Reformation. Seine Bibliothek umfasste 359 Bände

60 Alojz Jembrih: Divergenzen in der Sprachauffassung Primus Trubers und Stephan Konsuls in Ungnads „Bibelanstalt“. In: Rolf-Dieter Kluge: Ein Leben zwischen Laibach und Tübingen. Primus Truber und seine Zeit; Intentionen, Verlauf und Folgen der Reformation in Württemberg und Innerösterreich. München 1995, S. 457.

61 Ernst Benz: Hans von Ungnad und die Reformation unter den Südslawen. In: Zeitschrift für Kirchengeschichte 58 (1939), S. 387-475, hier S. 450.

62 Jakob Andreae: Dreyzehen Predigen vom Türcken. Tübingen 1568; vgl. dazu Siegfried Raeder: Die Türkenpredigten des Jakob Andreä. In: Brecht, Theologen und Theologie (wie Anm. 53), S. 96122.

63 Franz Brendle: Michael Tiffernus (1488-1555). Humanistischer Lehrer, politischer Ratgeber und Vertrauter Herzog Christophs von Württemberg, in: Lorenz, Primus Truber (wie Anm. 1), S. 229-246. 
und ging an das Tübinger Stift. Hier bildete sie den Grundstock der heute noch bestehenden Stiftsbibliothek. ${ }^{64}$ Der zweite Teil des Vermächtnisses, die als „Stipendium Tifferniticum“ oder einfach als „Tiffernum“ bezeichnete Studienstiftung bestand aus einem Kapital von 2.320 Gulden, mit dem insgesamt vier Freistellen am Stift bezahlt wurden, die erstmals auch Ausländern offenstanden und ihnen ein kostenfreies Studium gewährten. ${ }^{65}$

Die Geschichte der Reformation ist somit auch eine Geschichte der Beziehungen zwischen Württemberg und Slowenien. ${ }^{66}$ Zahlreiche slowenische Gelehrte, vertrieben von der Gegenreformation in ihrem Heimatland, wirkten im protestantisch gewordenen Herzogtum im Südwesten des Reiches. Von hier aus trugen sie wiederum als Übersetzer, Buchdrucker und Theologen maßgeblich zur Reformation in ihrem Heimatland bei. So kann gerade für die Reformationsentwicklung Südosteuropas ein spezifisch württembergischer Einfluss geltend gemacht werden, der sich in erster Linie mit dem Reformator Sloweniens, Primus Truber, verbindet.

64 Martin Brecht: Die Entwicklung der Alten Bibliothek des Tübinger Stifts in ihrem theologieund geisteswissenschaftlichen Zusammenhang, in: BWKG 63 (1963), S. 3-103.

65 Vgl. auch Otto Schmoller: Die Anfänge des Theologischen Stipendiums („Stifts“) in Tübingen unter Herzog Ulrich 1536 bis 1550. Stuttgart 1893; Martin Leube: Die Stiftung Tifferns beim Tübinger Stift. In: BWKG 23 (1919), S. 171-174.

66 Matthias Murko: Die Bedeutung der Reformation und Gegenreformation für das geistige Leben der Südslaven. Prag/Heidelberg 1927; Günther Stökl: Die deutsch-slavische Südostgrenze des Reiches im 16. Jahrhundert. Breslau 1940; Rudolf Trofenik (Hg.): Abhandlungen über die slowenische Reformation. München 1968; Rolf-Dieter Kluge: Frühe Tübinger Beiträge zum Verlauf und zur Erforschung der slowenischen Reformation. In: 16. stoletje v slovenskem jeziku, književnosti in kulturi. Ljubljana 1986, S. 209-226. 